\title{
Wearable Device for Monitoring Respiratory Phases Based on Breathing Sound and Chest Movement
}

\author{
Yusuke YuASA, , " Kenji SuzUKI ${ }^{* *}$
}

\begin{abstract}
Asthma is a chronic respiratory disease, in which symptoms appear or intensify suddenly, even when patients are being monitored by doctors. Continuous measurement is important to monitor a patient's breathing without missing asthma attacks. In this study, we propose a method of continuous breathing monitoring in daily life using a wearable device. There are several studies using microphones to continuously monitor breathing during activities, which show various possibilities of extracting qualitative characteristics related to asthma. Other studies on breathing measurement using accelerometers or belts have achieved breathing detection and measurement without ambient acoustic noises. Taking advantage of the breathing sound and chest movement signals, they are simultaneously acquired using a chest-mounted device, which consists of a microphone, a photoreflector, and a flexible cover. Various acoustic noises and body movements are present in the environment. Thus, acquiring these two different signals in a complementary manner makes it easier to detect breathing in daily life. For monitoring asthma, we focused on detecting the breathing phases. Most of the asthma symptoms appear during the exhalation phase. Thus, phase detection plays an important role as an asthma symptom identifier. We developed a new algorithm for breathing phase measurement using both acquired signals. The algorithm is based on the periodicity of the chest movement signal. Breathing sounds are analyzed considering their frequency characteristics. In this paper, the basic performance of the proposed device in an experimental condition which is quiet and without participant movements is examined. The results of performance evaluation confirm that the left medial side of the second intercostal space is appropriate for placing the device and studying the correlation between breathing sound amplitude and tidal volume, which implies a potential to acquire tidal volumes. The phase measurement experiment shows that chest movement can be used for estimating the breathing period. The portable system developed can measure breathing in external conditions and tracking the wearer's location. Making the system portable expands the measurable situations and facilitates an acquisition of time and location information, which is useful in identifying the causes of asthma attacks.
\end{abstract}

Keywords: breathing measurement, breathing phases, asthma monitoring, wearable device.

Adv Biomed Eng. 8: pp. 85-91, 2019.

\section{Introduction}

Long-term vital measurement in daily life is important for patients with chronic disease and facilitates follow-up of such conditions. Breathing measurement is a vital examination for asthma. Asthma symptoms, called "asthma

This study was presented at the Symposium on Biomedical Engineering 2018, Nagoya, September, 2018.

Received on July 23, 2018; revised on October 10, 2018 and January 22, 2019; accepted on January 23, 2019.

* Graduate School of Systems and Information Engineering, University of Tsukuba, Tsukuba, Japan.

** Center for Cybernics Research, University of Tsukuba, Tsukuba, Japan.

\# 1-1-1, Tennodai, Tsukuba, Ibaraki 305-8573, Japan.

E-mail: yusuke@ai.iit.tsukuba.ac.jp attack", may appear or aggravate suddenly, even when the patients are with their doctors or families. To monitor a patient's breathing without missing asthma attacks, continuous measurement is useful and is performed using wearable devices.

Wearable devices that generate breathing-related biomedical signals have been developed on a large scale [1]. Breathing sound and chest movement are a part of such biomedical signals. Breathing sound measured by microphones over human skin includes many asthma-related characteristics such as breathing rate, wheeze [2, 3], tidal volume [4], flow rate [5], and cough [6]. Moreover, chest movement signals can be acquired using a belt-shaped device [7] or an accelerometer [8]. This signal provides robust breathing detection because it shows the simple periodic characteristics to- 
gether with the breathing cycle without being affected by the ambient acoustic noise.

Considering the advantages of breathing sound and chest movement signals, we previously proposed a wearable device that measures both of these signals simultaneously for asthma monitoring in a daily living environment [9]. The proposed device, which is smaller than $35 \mathrm{~mm}$ in diameter, does not interrupt the airflow and does not restrain the patient's chest. Thus, it does not affect the patient's breathing ability. Hence, it is a suitable method for prolonged monitoring in daily life. Furthermore, clear respiratory phases are required for identifying asthma symptoms. Although phase measurement methods were studied $[10,11]$, these studies were conducted without considering daily life environment. Thus, as the first step of breathing measurement in daily life, the feasibility of respiratory phase measurement using the wearable device was examined in our previous work [9]. In the present study, we propose a method for breathing phase measurement that includes a more accurate processing algorithm. We also demonstrate the performance of phase measurement and discuss it in terms of accuracy in subjects.

There are many causes of asthma attacks, such as allergens, climate, and polluted air, and the type and amount of risk factors differ according to the location and time. In addition, the factors that affect the condition depends on the patient. Therefore, patient's location and time measurements are also required to assist doctors in making a diagnosis. Recently, smartphone-based health monitoring in different locations has been studied [12], and breathing measurement using smartphones has also been conducted [13]. A smartphone acquires the GPS and time data, which can be used for detecting the location along with breathing measurement.

A conceptual diagram of asthma monitoring, which this study aims to achieve with the portable breathing measurement system, is shown in Fig. 1. To measure the breathing phases in a living environment, we developed

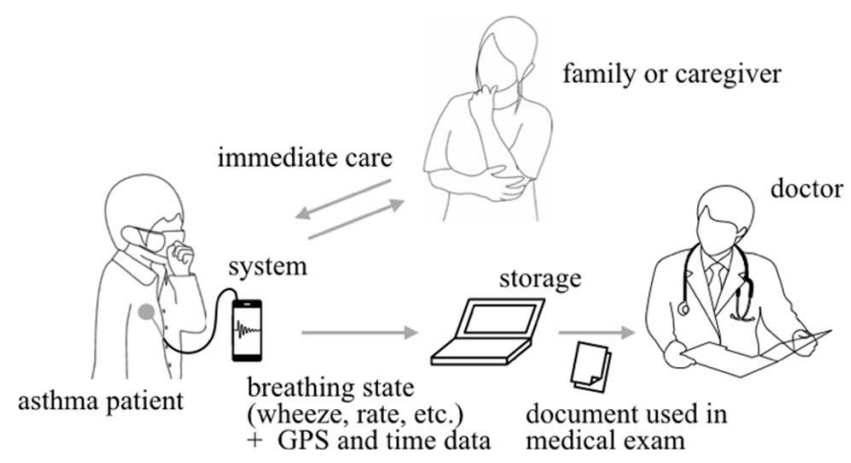

Fig. 1 Conceptual diagram of asthma monitoring in daily life using the proposed system. a new algorithm, based on a simple periodicity of the chest movement, and evaluated its performance in participants. In addition, we developed a portable system using a smartphone for acquiring the wearer's location and time data, together with a wearable device for breathing measurement. We also performed a demonstration experiment of the portable system for location measurement.

\section{System overview}

Figure 2 shows the appearance of the developed device called hybrid-based aspiration and respiration sensing (HARS) [9]. Both breathing sounds and chest movements are acquired by the HARS. The device has an elastic flexible cover (TangoBlackPlus, Stratasys Co.), a microphone (MEMS Microphone Breakout - INMP401, Sparkfun Co.), and a photoreflector (SG-2BC, Kodenshi Co.) that measures the density of the reflected infrared light. It is attached to the chest using a ring-shaped adhesive gel sheet, and the air between the device and chest skin is removed by pushing the cover.

\subsection{Breathing signal acquisition}

Breathing sounds from the chest wall are obtained by a microphone located in the space between the HARS device and the chest skin. The signal is filtered with a bandpass filter of $65-1,000 \mathrm{~Hz}$ and acquired at a sampling frequency of $44.1 \mathrm{kHz}$.

\subsection{Chest movement signal acquisition}

The chest movements are measured by the photoreflector utilizing the flexibility of the cover. The anterior ribs rise with inhalation, causing a forward anterior chest movement. On the contrary, the ribs descend with exhalation and move the anterior chest backward. The rib movement changes the horizontal section area of the chest. During inhalation, the cover of the HARS attached to the upper chest is pulled and deformed by the skin accompa- (a)

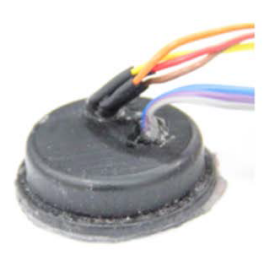

(b)

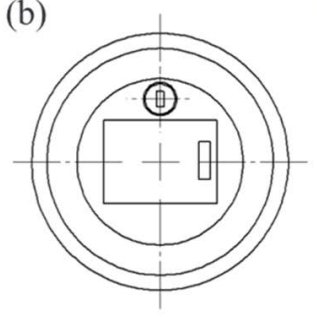

(c)

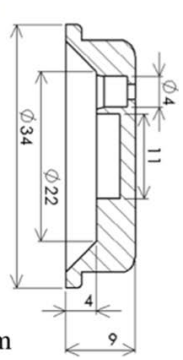

Fig. 2 (a) Appearance of the device called HARS, (b) the cover viewed from the bottom, and (c) cross section of the cover with pockets for installing a microphone and a photoreflector. 
nying expansion of the chest section. Furthermore, the angle of the photoreflector, density of the reflected infrared light, and sensor value change. During exhalation, the density of the reflected light and sensor value change in a reverse manner because of the inverse deformation. These changes in sensor value are measured as chest movements. An illustrative figure of the changes in photoreflector value and the deformations caused due to breathing are shown in Fig. 3. The signal is low-pass filtered at a cutoff frequency of $5 \mathrm{~Hz}$, and acquired at a sampling frequency of $10 \mathrm{~Hz}$.

\subsection{Algorithm for phase measurement}

The proposed digital signal processing flow for respiratory phase measurement is shown in Fig. 4. First, the acquired breathing sound is filtered with a Butterworth band-pass filter of $150-400 \mathrm{~Hz}$. The purpose of filtering with the narrow frequency band is to measure breathing phases. Therefore, wider sound frequency bands can be used for measuring other characteristics such as wheezing sounds. The signal power in this frequency band increases during breathing and the band does not include the low-frequency band that is affected by the main utility frequency and heart sounds. Wheezing sounds are reported to be in the wide frequency band of $100 \mathrm{~Hz}$ to $1 \mathrm{kHz}$ [14]. Thus, the signal power in the pass band may have a large value for asthma patients. Subsequently, the band-pass filtered signals are filtered with a Hampel fil-

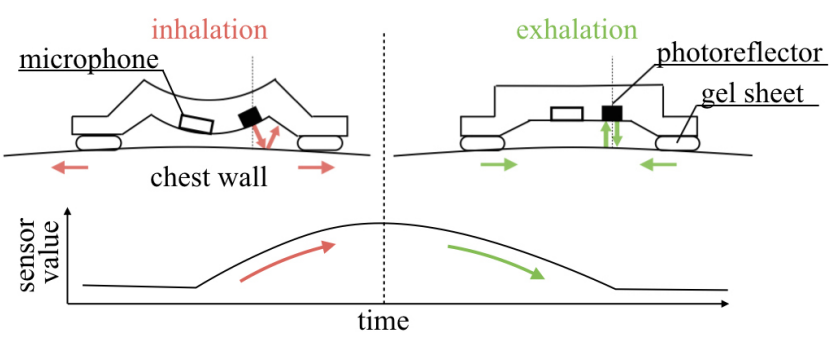

Fig. 3 Sensor design and illustrative chest movement curve acquired based on deformation of HARS. ter having a window length of $0.1 \mathrm{~s}$. Thereafter, the signals undergo outlier removal, full-wave rectification, Butterworth low-pass filtering at a cutoff frequency of $2 \mathrm{~Hz}$, and normalization. Moreover, the chest movement signal is smoothened with a Gaussian weighted moving average filter having a window length of $1.5 \mathrm{~s}$ and normalized later. Let $u_{s}$ and $u_{c}$ be the smoothed sound and movement signals, respectively. Given that the angle of the attached HARS can affect the direction of the sensor value changes, the first set of inhalation and exhalation after starting the measurement is used for calibration. This process is used to allow the movement signals to increase and decrease corresponding to inhalation and exhalation, respectively. The peak detection algorithm is applied to $u_{s}(t)$ and also to $u_{c}(t)$. Moreover, $t_{s}(k)$ and $t_{c}(k)$ are defined as the time of the $k$ th peak, which have prominences of at least 0.1. As shown in Fig. 5, the prominence of the $k$ th peak in $u_{s}(t)$ is defined as the amplitude difference from a foot point $u_{s}\left(t_{f}\right)$ to the peak value $u_{s}\left(t_{s}(k)\right)$. Two minimum points, before and after the peak, are detected within the nearest points $t_{b}(k), t_{a}(k)\left(t_{b}(k)<\right.$ $\left.t_{a}(k)\right)$, such that $u_{s}\left(t_{b}(k)\right)=u_{s}\left(t_{a}(k)\right)=u_{s}\left(t_{s}(k)\right)$. The time that has a higher minima is selected as $t_{f}$. The prominence in $u_{c}(t)$ is determined using the same algorithm. The middle point between $t_{c}(k)$ and $t_{c}(k+1)$ is interpolated if the interval is twice or longer than the median of all intervals, and $t_{c}(k)$ is eliminated if the interval is half or less than the median.

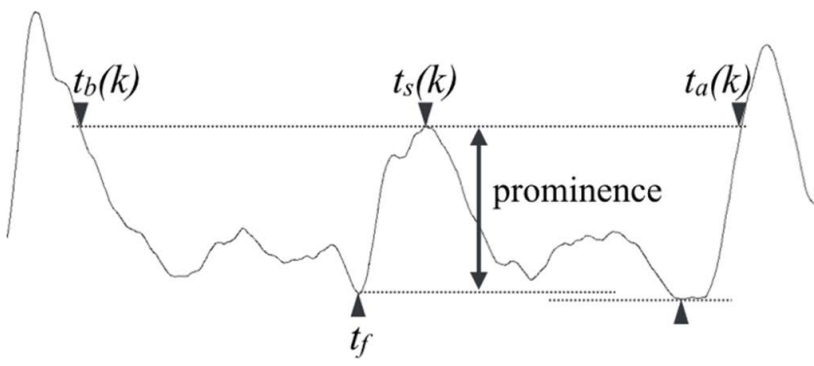

Fig. 5 Illustration of the signal $u_{s}(t)$ and the definition of the prominence of the peak at $t_{s}(k)$. (a)

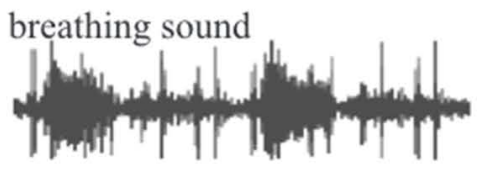

chest movement

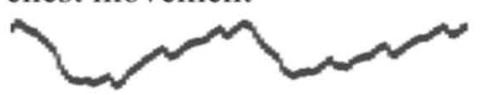

(b)

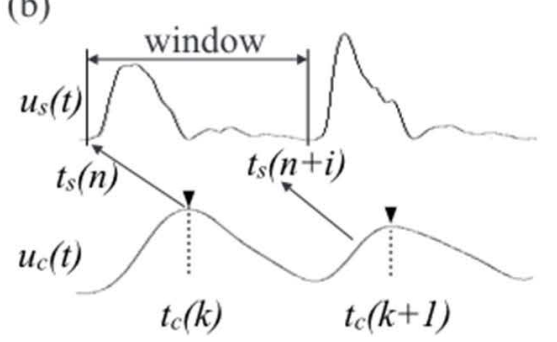

(c)

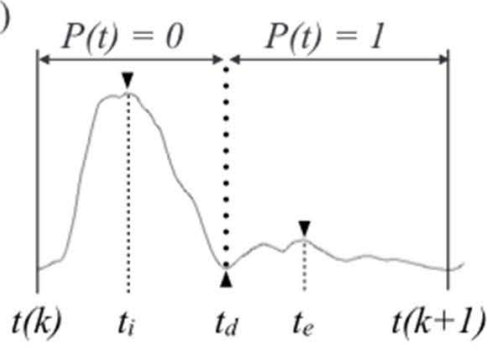

Fig. 4 Signal processing for breathing phase measurement. (a) Raw breathing sound and chest movement signal, (b) extracting a time window corresponding to an estimated set of inhalation and exhalation, and (c) discriminating an inhalation and exhalation term. 
The simple periodic characteristics of the chest movement signal are used to estimate the period of breathing. $t_{c}(k)$ corresponds to the end time of inhalation, and the interval between the two peaks represent each breathing cycle that includes one inhalation and one exhalation. Therefore, time $t(k)$, which separates the cycles, is selected using a constant $\tau$ as follows:

$$
\begin{gathered}
t(k)=\underset{t_{s}(n)}{\arg \min }\left|t_{c}(k)-\tau-t_{s}(n)\right|, \\
\tau=\frac{1}{2 M} \sum_{k=1}^{M-1}\left(t_{c}(k+1)-t_{c}(k)\right) .
\end{gathered}
$$

where $M$ is the length of $t_{c}(k)$. The interval between $t(k)$ and $t(k+1)$ is determined as the $k$ th window, which has one breathing cycle (Fig. 4b). The peak detection algorithm is applied to $u_{s}(t)$ within each window. Let $t_{i}$ and $t_{e}$ be the time of the first and last peaks. respectively, which have prominences of 0.05 . The time $t_{d}$ within $t_{i}$ and $t_{e}$ is estimated as the separation point of inhalation and exhalation, and represented as follows:

$$
t_{d}=\arg \min u_{s}(t), \quad\left(t_{i}<t<t_{e}\right)
$$

Finally, the breathing phase $p(t)$, which has a binary value corresponding to each respiratory phase, is computed as follows:

$$
p(t)= \begin{cases}0 & \left(t(k)<t \leq t_{d}\right) \\ 1 & \left(t_{d}<t<t(k+1)\right)\end{cases}
$$

where $p(t)=0$ corresponds to inhalation and $p(t)=1$ corresponds to exhalation (Fig. $\mathbf{4 c}$ ). The detection of $t_{i}, t_{e}, t_{d}$, and $p(t)$ is conducted for all estimated windows.

\section{Performance evaluation}

To verify the feasibility of the HARS, experiments were conducted in healthy participants. During recordings using HARS, the participants were instructed to stay in a sitting position, pinch their noses, and hold the mouthpiece of the spirometer (Chestgraph HI-301 U, made by Chest Co.) in the mouth. The ground truth of breathing terms and breathing phases was obtained from the graph of the spirometer. The actual breathing terms were visually detected by defining inflection points of the graph as boundaries between two phases. The actual phases were classified into inhalation and exhalation by visually confirming the gradients. The sound and chest movement signals were used as input values into the line-in jack and USB serial port on a laptop PC, and signal processing was conducted using MATLAB (MathWorks Inc.). This experiment was reviewed and approved by the Institutional Ethical Committee at the University of Tsukuba (2017R170).

\subsection{Device position and signal characteristic}

To verify the robustness of the device position for operators without medical knowledge, and the ability to ac- quire quantitative characteristics of breathing sounds, the amplitude characteristics of the signals acquired using HARS at different placement positions and at different tidal volumes were studied.

As shown in Fig. 6, our proposed device position at left medial side of the second intercostal space (which is generally selected during the auscultation for estimating breathing state) was compared with nearby positions at the first and third intercostal spaces. The data recorded at each position included 100 sets of breathing phases from 10 healthy male participants in their twenties. The participants were asked to look at the graph of the spirometer and breathe at a tidal volume of $1,500 \mathrm{~mL}$. The results of the comparison of device placement positions are shown in Fig. 7. The acoustic energy was calculated by integrating the sound signals over the breathing cycle and aver-

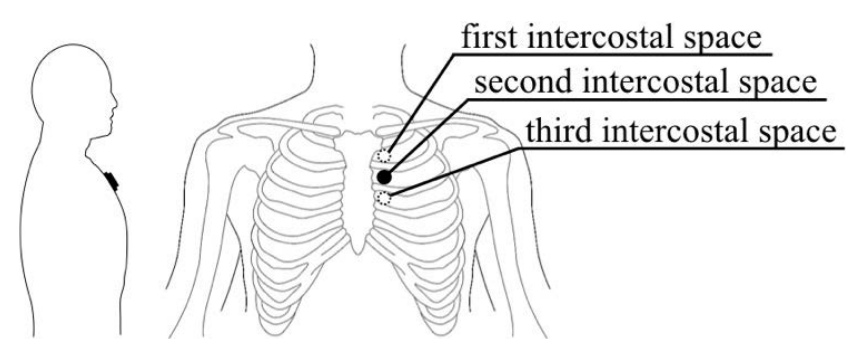

Fig. 6 Device position viewed from side and vertical positions.
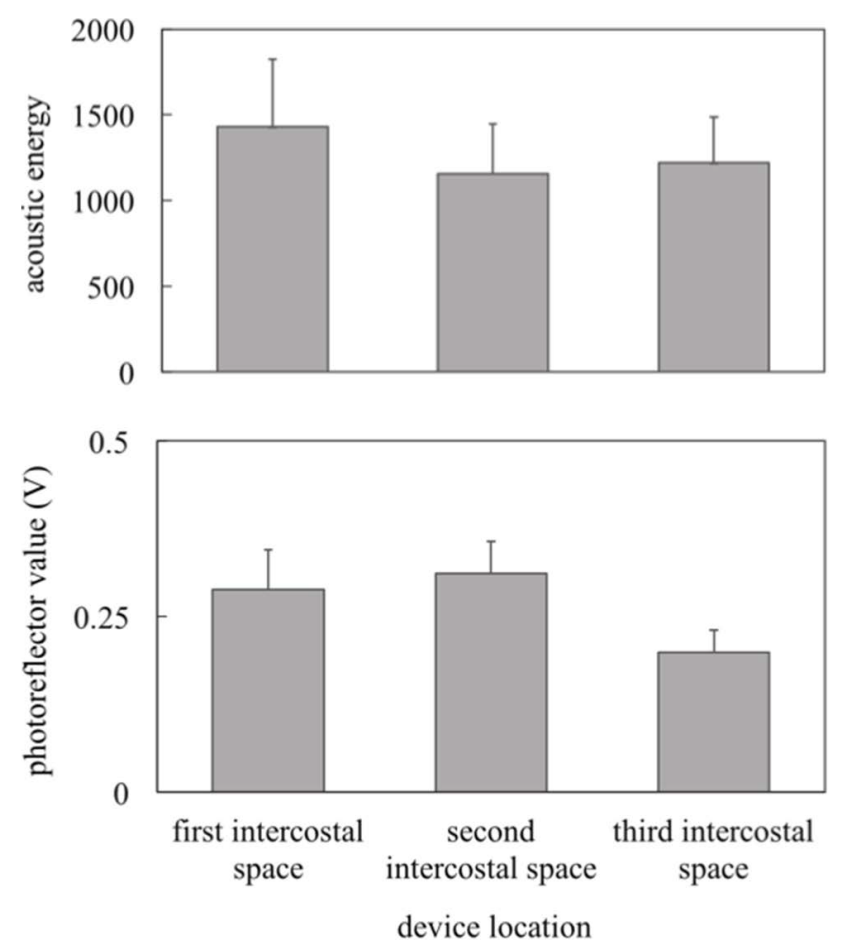

Fig. 7 Acoustic energy integrated over 10 breathing cycles, and the photoreflector value at each location. 
aging the integration of 10 breathing cycles. The photoreflector value was the mean difference between the maximum and minimum values within the breathing cycle.

This result confirms that the device is able to acquire sound signals with large amplitudes on the upper chest, around the first intercostal space. This finding is known from a report on vesicular lung sound amplitude [15], which describes that airflow going through the respiratory tract generates more sound over the upper airway than the bottom, and the region where the upper airway is the closest to the skin is located around the upper chest. We suspect that the small difference between the values around the second and the third intercostal space may be caused by heart sounds that have higher amplitudes on average than the lung sounds in the acquired signals. To examine whether there is any difference between the proposed position and other positions, Steel-Dwass test was used to analyze the results of the sound. Test Statistics of the proposed location versus the first and third intercostal space were 1.4 and 0.1 , respectively. For a significance level of $5 \%$, the corresponding test statistic was 2.34. Because test statistics of the breathing sound analysis was smaller than 2.34 , there was no significant difference.

Data of chest movement were analyzed by SteelDwass test as for sound. The test statistics of the proposed position versus the first and third intercostal spaces were 1.4 and 4.8, respectively. Based on these results, the amplitudes were significantly different between the proposed position and the third intercostal space. Cohen's $\mathrm{d}$ of data at the proposed and lower locations was 0.76 , which was high in general. Therefore, the third intercostal space was not the preferred position.

Even though the first intercostal space is around $2-3 \mathrm{~cm}$ above the proposed location, close to the collar bone, chest movement signal may be affected by shoulder movements. Amplitude characteristics remain similar to those around the proposed position. As a result, the left medial side of the second intercostal space is the preferred position for the HARS, while a position around $2-3 \mathrm{~cm}$ higher than the preferred position can still be used. Such position robustness leads to high usability.

The breathing sounds at different tidal volumes of $1,000,1,500$, and $2,000 \mathrm{~mL}$ were also obtained. This data included 100 breathings acquired for each tidal volume from the same participants. The amplitude characteristics of sounds at different volumes are shown in Fig. 8. Consequently, the correlation between tidal volume and acoustic energy was confirmed. These findings indicate that HARS is able to acquire quantitative characteristics of breathing and detect asthma-related characteristics.

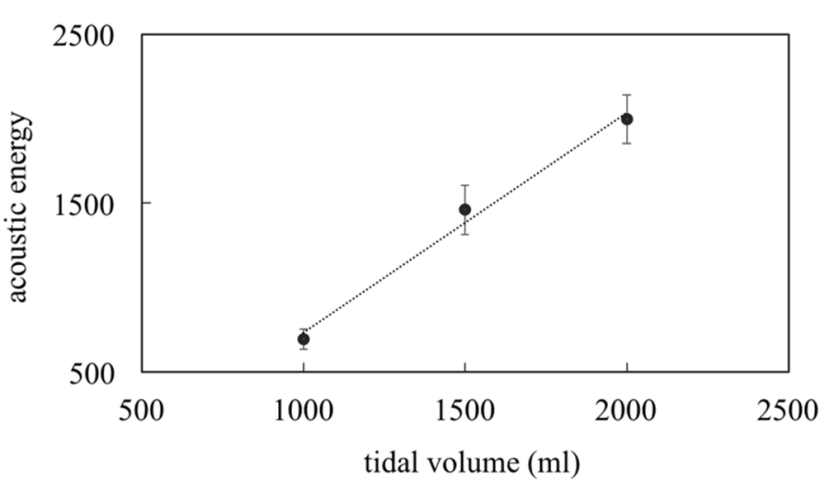

Fig. 8 Acoustic energy integrated over 10 breathing cycles at each tidal volume.

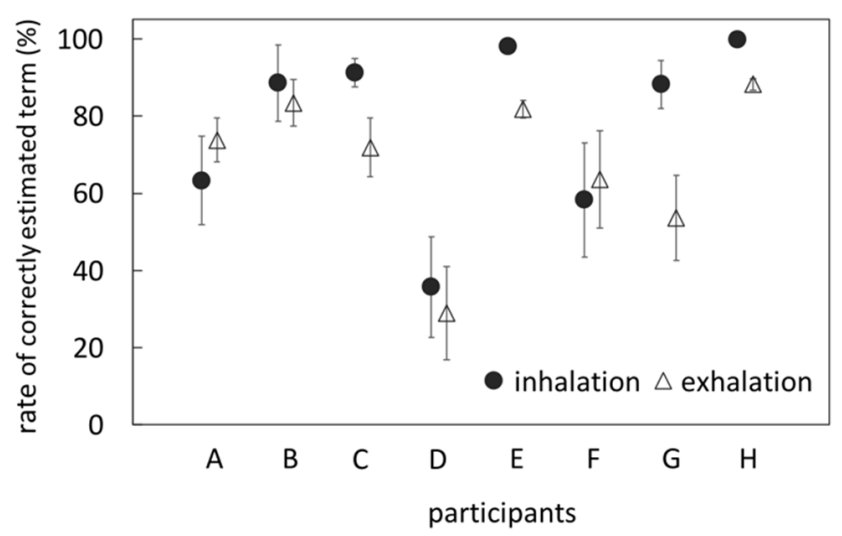

Fig. 9 Rate of correctly estimated inhalation and exhalation term for each participant.

\subsection{Phase measurement}

To evaluate the performance of the respiratory phase detection algorithm, the proposed signal processing method was used to analyze the signals. The data analyzed contained 80 sets of breathing phases recorded over the second intercostal space from eight participants who were all males in their twenties. The agreement rates of the estimated breathing phases are shown in Fig. 9. Correctly detected term was defined as $60 \%$ or higher agreement between the estimated term and the actual breathing term. The mean accuracy rates of the detected breathing, identified inhalation, and identified exhalation were $65.6 \%, 62.5 \%$, and $61.3 \%$, respectively. These results show that the signal processing method proposed in this paper is more accurate than the method proposed in our previous work [9] that had a maximum accuracy of $50 \%$. These results show that chest movements are more useful for stable measurement of breathing periods than breathing sounds. All breathing phases were detected and the phases were correctly identified in some participants, whereas the detection rates were $40 \%$ or less in others. A possible reason of the low detection rate is the 
small breathing sounds in some participants. The breathing sound amplitude was relatively small; especially in participants with large BMI, the $t_{s}(k), t_{i}$, and $t_{e}$ were difficult to detect. A negative correlation is shown between BMI and acoustic energy with a correlation coefficient of -0.55 . Hence, it is conceivable that thick fat or muscle decreases the breathing sound amplitude. In addition, low signal-to-noise ratio also decreases a number of detected peaks. Therefore, the accuracy of detection may be improved by enhancing the sensitivity of the microphone. Furthermore, to reduce the noise accompanying enhanced sensitivity, use of a device cover with sound absorbing pattern on the surface or a cover with acoustic absorbent material may be useful. In addition, sudden baseline variation of chest movements also resulted in low accuracy in one participant. The possible cause of this is insufficient gel sheet sliding. Thus, we plan to re-consider the device pasting material. To increase the accuracy of phase estimation, a method of extracting confident intervals may be used. The confident interval is the interval in which the wearer is not moving excessively and the device is working stably. However, the high accuracy of some results shows that identification of the periodicity of chest movement is useful for breathing detection.

\section{Demonstration experiment}

To detect the patient's location and identify the main risk factors, we developed a portable recording system that also provides time and the GPS data, using an Android smartphone (Nexus 5, LG Electronics). A participant who was a healthy male in his twenties was instructed to breathe deeply 15 times per minute in a standing posture. The breathing sounds and chest movements were recorded for one minute at four locations during the afternoon. There were some people walking but no talking voices around the locations of measurement. Breathing was detected by the proposed algorithm and the breathing rate was computed by dividing the number of breathing detected by the recording duration $(60 \mathrm{~s})$. The estimated breathing rates and end times of recordings were plotted on the map with the locations where each recording was acquired. The result is shown in Fig. 10. The estimated breathing rate was only 13 in locations $\mathrm{C}$ and $\mathrm{D}$ because of sudden variations of baseline chest movement signal. A possible reason is the low adhesiveness of the gel sheet. The minimum distance between any two locations was approximately $50 \mathrm{~m}$, which were clearly distinguished. Consequently, this experiment shows that the proposed system has the potential of performing breathing measurements in external conditions and detection of the wearer's location.

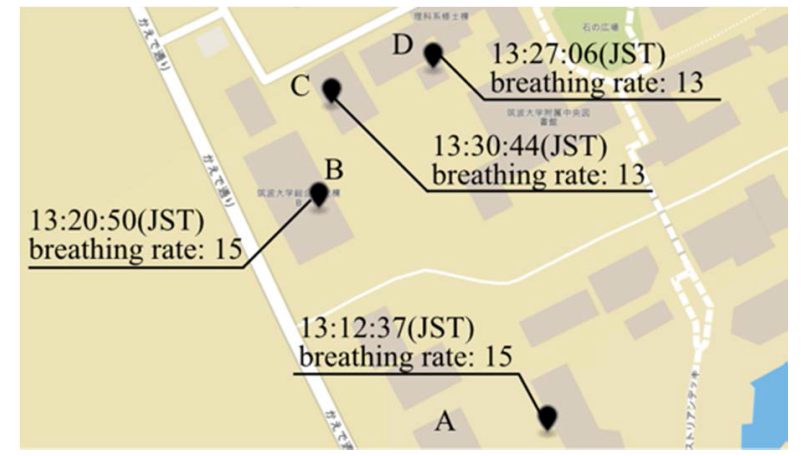

Fig. 10 Map with recording time, locations, and estimated breathing rates made by using HARS and a smartphone-based recording system.

\section{Conclusion}

In this study, we introduced a wearable device for simultaneous measurements of breathing sound and chest movement in daily life. The device is attached on the upper chest with an adhesive gel sheet and measures both signals in an area smaller than a circle of $35 \mathrm{~mm}$ diameter. The chest movement signal is acquired by a photoreflector using the deformation of the flexible cover accompanying the expansion and contraction of the respiring chest. In this report, we propose a modified signal processing method for phase measurement based on chest movement that has simple periodicity and corresponds to the wearer's body activities only. To examine the characteristics of acquired signals and evaluate the proposed algorithm for phase detection, we performed experiments in a quiet environment without participant movements. According to the experiments, the left medial side of the second intercostal space is the preferred position for placing the device because the signal amplitudes at that location are large and possibly less affected by shoulder movements. In addition, a correlation between sound amplitude and tidal volume was observed. The breathing phase identification experiment shows that the periodicity of the chest movements can be used to estimate the breathing periods and phases. The frequency characteristics change readily depending on the wearer's condition such as wheezing. Thus, a phase measurement algorithm that relies on amplitude more than frequency characteristics is useful in situations in which the device is intended to be used. The demonstration experiment shows the possibility of acquiring breathing measurement in external conditions together with location information using a smartphone. Furthermore, the possibility of smartphones to monitor air pollution with several wearable devices has been reported [1]. Thus, the proposed portable system has a potential to be further enhanced as an identifier for causes of asthma in individ- 
uals. Moreover, the number of smartphone users is increasing. Thus, a system with a smartphone can be used with greater ease than any special recording device. These results do not show the performance of the device in daily living environments. Therefore, we plan to investigate the feasibility of using the proposed method in other situations such as during light exercises in the future.

\section{Acknowledgment}

The authors would like to thank Baptiste Bourreau for developing the smartphone application used for recording signals, time, and GPS data.

\section{Conflict of Interest}

We have no conflict of interest relationship with any companies or commercial organizations based on the definition of the Japanese Society for Medical and Biological Engineering.

\section{References}

1. Aliverti A: Wearable technology: role in respiratory health and disease. Breathe. 13(2), pp. e27-e36, 2017.

2. Zhang J, Ser W, Yu J, Zhang TT: A novel wheeze detection method for wearable monitoring systems. IUCE. pp. 331-334, 2009.

3. Taplidou SA, Hadjileontiadis LJ, Kitsas IK, Panoulas KI, Penzel T, Gross V, Panas VM: On applying continuous wavelet transform in wheeze analysis. Conf Proc IEEE Eng Med Biol Soc. pp. 3832-3835, 2004.

4. Chen G, de la Cruz I, Rodriguez-Villegas E: Automatic lung tidal volumes estimation from tracheal sounds. Conf Proc IEEE Eng Med Biol Soc. pp. 1497-1500, 2014.

5. Yadollahi A, Moussavi ZM: A robust method for estimating respiratory flow using tracheal sounds entropy. IEEE Trans Biomed Eng. 53(4), pp. 662-668, 2006.

6. Rahman T, Adams AT, Zhang M, Cherry E, Zhou B, Peng H, Choudhury T: BodyBeat: A mobile system for sensing nonspeech body sounds. MobiSys '14. pp. 2-13, 2014.

7. Anusha AR, Soodi AL, Kumar SP: Design of low-cost hardware for lung sound acquisition and determination of inspiratory-expiratory phase using respiratory waveform. ICCCNT. pp. 1-5, 2012.

8. Bates A, Ling MJ, Mann J, Arvind DK: Respiratory rate and flow waveform estimation from tri-axial accelerometer data. BSN. pp. 144-150, 2010.

9. Yuasa Y, Takahashi K, Suzuki K: Wearable flexible device for the respiratory phase measurement based on sound and chest movement. SMC. pp. 2378-2383, 2017.

10. Jin F, Sattar F: Unsupervised phase detection for respiratory sounds using improved scale-space features. ISSPIT. pp. 97-102, 2016.

11. Huq S, Moussavi Z: Automatic breath phase detection using only tracheal sounds. Conf Proc IEEE Eng Med Biol Soc. 50(3), pp. 272-275, 2010.

12. Guerrache F, Aldabbagh A, Kanjo E: Multiple sensor fusion approach to map environmental noise impact on health. UbiComp
'16. pp. 1074-1078, 2016.

13. Sohn K, Merchant FM, Sayadi O, Puppala D, Doddamani R, Sahani A, Singh JP, Heist EK, Isselbacher EM, Armoundas AA: A novel point-of-care smartphone based system for monitoring the cardiac and respiratory systems. Sci Rep. 7, 44946, 2017.

14. Gurung A, Scrafford CG, Tielsch JM, Levine OS, Checkley W: Computerized lung sound analysis as diagnostic aid for the detection of abnormal lung sounds: a systematic review and meta-analysis. Resp Med. 105(9), pp. 1396-1403, 2011.

15. Dennis MO, Kraman S: Vesicular lung sound amplitude mapping by automated flow-gated phonopneumography. J Appl Physiol Respir Environ Exerc Physiol. 53(3), pp. 603-609, 1982.

\section{Yusuke Yuasa}

Yusuke YUASA received B.E. degree from University of Tsukuba, Japan, in 2017. He is currently a student at Master's Program in Intelligent Interaction Technologies at University of Tsukuba. His research interests include biomedical engineering, signal processing, and wearable device.

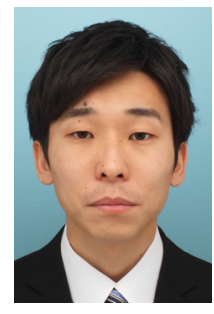

\section{Kenji Suzuki}

Kenji Suzuki is currently a Full Professor of the Faculty of Engineering, Information and Systems, and at the Center for Cybernics Research. He received the B.S. in Physics, M.E. and Dr. Eng. in Pure and Applied Physics from Waseda University, Tokyo, Japan, in 1997, 2000 and 2003, respective-

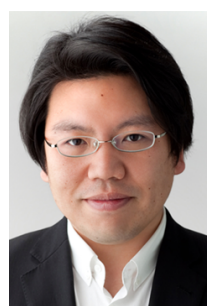
ly. Prior to joining the University of Tsukuba in 2005, he was a Research Associate with the Dept. of Applied Physics, Waseda University. He was a visiting researcher at the Laboratory of Musical Information, University of Genoa, Italy, and also at the College de France, Paris. He is a member of IEEE and ACM. 